\title{
Network Security: Hybrid IDPS
}

\author{
Youssef Senhaji \\ Architecture System Team \\ Hassan II University of Casablanca ENSEM \\ Casablanca, Morocco
}

\author{
Hicham Medromi \\ Architecture System Team \\ Hassan II University of Casablanca ENSEM \\ Casablanca, Morocco
}

\begin{abstract}
This paper deals with the issue of computer security, which aims to develop a robust and independent security architecture. This architecture consists of several probes spatially distributed to several locations in the network (sensitive servers, DMZ, workstations, etc.). These probes are NIDPS, HIDPS, KIDPS and Arduino Yun Board. These same probes were semantically distributed according to three threat detection methods. At the end of this paper, we developed a hybrid system consisting of a software IDPS represented by a probe developed under Visual $\mathrm{C}++$ and an embedded solution developed under Python in an Arduino YUN board. We carry out a series of computer attacks on our detection system to assess its response time.
\end{abstract}

\section{General Terms}

Network Security, IDPS, Real Time, Embedded System, Distributed System, Arduino.

\section{Keywords}

Network Security, IDPS, Real Time, Embedded System, Distributed System, Arduino.

\section{INTRODUCTION}

IDPS are important computer network security system.

In this paper we will present a combination of two IDPS configuration. The first configuration is a software solution developed with Visual $\mathrm{C}++$.

The second configuration is a hardware proposal embedded in an Arduino Yun board.

On these systems, we will make several computer attacks to see their reactions.

But before we begin, we'll introduce the concepts: detection method and distributed system and then we'll present the Arduino Yun Board.

\section{COMMON DETECTION METHODS}

Intrusion detection is the process of monitoring the events occurring in a computer system or network and analyzing them for signs of possible incidents, which are violations or imminent threats of violation of computer security policies, acceptable use policies, or standard security practices. [1]

Among the detection methods used by IDPS, we find:

- Signature Based Detection: this method is based on the comparison of the units of activities (Package, Log Entry) to a list of models by using the operators of comparison. A model corresponds to a known threat.

- Anomaly Based Detection: It is a method basing itself on statistical calculations and it has a "Profile" which represents the normal behavior. So this method consists of making comparison between the events and the definition of the events considered normal to detect deviations.

- Stateful Protocol Analysis: This method compares the protocols and their profiles. In addition, it exploits the combination of the request and its answer to be able to evaluate the state.

\section{DISTRIBUTED SYSTEM}

A Distributed system can be distributed based on an existing conceptual distance between its components.

This distance can be:

- Spatial: distribution by different processes assigned to solve a problem related to space.

- Semantic: distribution by the specificity of knowledge and a particular know-how.

- Structural: representations are heterogeneous and reasoning mechanisms are different.

- Semantic: according to its function and its role within the system.

\section{ARDUINO YUN BOARD}

The Arduino Yun is an electronic board that uses the Atmel processor ATmega32U4. Besides of that, it has an additional processor: Atheros AR9331, that turn the Linux distribution OpenWrt Linino.

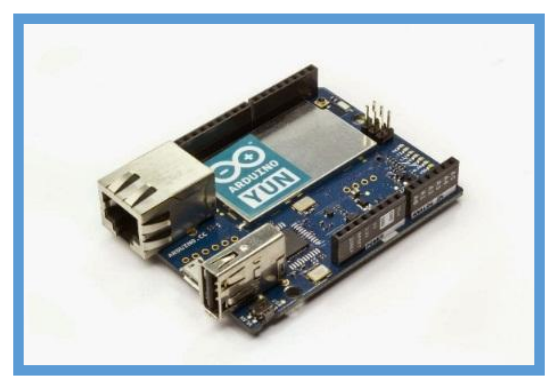

Fig 1: Arduino Yun Board

\section{PROPOSED ARCHITECTURE}

\subsection{Introduction}

Prior to deployment of the security solution, we assume that users are aware of the importance of security and its challenges and that all systems and applications are constantly updated (security patches).

Suppose we have a network with the following elements:

- A LAN (local area network): consists of several workstations. 
- A DMZ (demilitarized zone): Consisting of machines on the internal network that need to be accessible from the outside (mail server, FTP server, web server ...)

- A Web Client: consists of Outside Network

\subsection{Spatial Distribution}

To secure the network while focusing on the concept of load reduction and increased response time, the security system will be deployed and distributed spatially in the network. It will be composed of several distributed software IDPS (hereinafter referred IDPS) and hardware embedded Arduino IDPS sensors (hereinafter referred ARD). And for a more reduction of the data loading on these sensors, they must be accompanied by pre-filtering firewalls which analyze the data stream before capture. Moreover, and for a complementary security solution we will combine between NIDPS and HIDPS. HIDPS will be deployed on the machines in the DMZ and on important servers. We can also add KIDPS (K: Kernel) for sensitive machines. Below the list of probes that we will use:

- Ks: KIDPS sensor for sensitive servers

- Hs: HIDPS sensor for important servers

- N1: NIDPS sensor analyzing traffic between the internal network and the Internet

- N2: NIDPS sensor analyzing traffic between the internal network or DMZ and Internet (before the firewall for its protection)

- N3: NIDPS sensor analyzing traffic between the elements of the DMZ and Internet

- Hi: sensor for HIDPS servers in the DMZ

- $\quad$ ARD : Network Arduino sensor

\subsection{Semantic Distribution}

In this step, we proceed to a second distribution, a semantic one based on IDPS method detection. This distinction aims to specialize the IDPS.

Thus, each IDPS and ARD will be divided into three parts:

- IDPS-SPA: Based on the "Stateful Protocol Analysis" as a method of detection

- IDPS-ABD: Based on "Anomaly Based Detection" as a method of detection

- IDPS-SBD: Based on "Signature Based Detection" as a method of detection.

- ARD-SPA: Based on the "Stateful Protocol Analysis" as a method of detection

- ARD-ABD: Based on "Anomaly Based Detection" as a method of detection

- ARD-SBD: Based on "Signature Based Detection" as a method of detection.

\section{TEST RESULTS FOR THE HYBRID SYSTEM: IDPS/ ARD}

To achieve our simulation on our system, we have developed 3 Systems:
The first is an application developed with $\mathrm{C}++$ making the role of an IDPS exploiting the PCAP library.

The second is a Python script embedded in a Yun Arduino board and doing the role of an IDS by exploiting RAW socket.

The third system is an application that generates targeted intrusion attacks.

Thus, we will initially attack a system protected by the binomial HIDPSS and ARD and secondly the case of a system protected by the binomial NIDPSS and ARD.

\subsection{HIDPS/ARD System}

\subsubsection{Diagram of the simulation}

As a first step, we will pair an HIDPSS and an ARD as below:

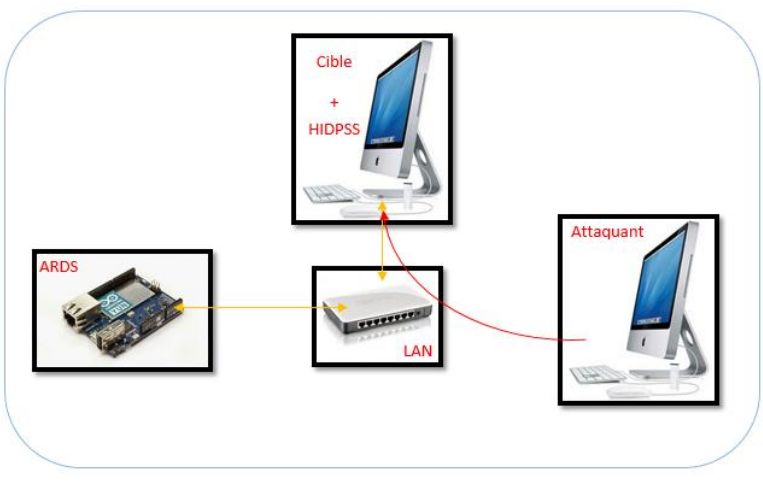

Fig 2: Case HIDPS/ARDS

\subsubsection{Evaluation of the detection time}

We carry out a series of attacks on our detection system to assess its response time to an attack. Thus we get the results below.

Table 1. Summary of different detection time - HIDPS / ARD

\begin{tabular}{|c|c|c|c|c|c|}
\hline $\begin{array}{l}\text { Attack } \\
\text { Number }\end{array}$ & $\begin{array}{l}\text { Attack } \\
\text { Instant }\end{array}$ & $\begin{array}{c}\text { ARD } \\
\text { Detection } \\
\text { Instant }\end{array}$ & $\begin{array}{c}\text { Detection } \\
\text { Time ARD } \\
\text { (ms) }\end{array}$ & $\begin{array}{c}\text { IDPS } \\
\text { Detection } \\
\text { Instant }\end{array}$ & $\begin{array}{c}\text { Detection } \\
\text { Time IDPS } \\
\text { (ms) }\end{array}$ \\
\hline 1 & $18: 11: 06,455$ & 18:11:11,317 & $0: 00: 04,862$ & 18:11:08,004 & $0: 00: 01,549$ \\
\hline 2 & $18: 11: 27,000$ & $18: 11: 35,347$ & $0: 00: 08,347$ & $18: 11: 28,300$ & $0: 00: 01,300$ \\
\hline 3 & 18:11:40,699 & 18:11:46,134 & $0: 00: 05,435$ & 18:11:43,011 & $0: 00: 02,312$ \\
\hline 4 & $18: 12: 03,000$ & $18: 12: 13,877$ & $0: 00: 10,877$ & 18:12:05,350 & $0: 00: 02,350$ \\
\hline 5 & $18: 12: 15,613$ & Not Detected & & $18: 12: 17,518$ & 0:00:01,905 \\
\hline 6 & $18: 12: 32,073$ & $18: 12: 42,245$ & $0: 00: 10,172$ & $18: 12: 33,758$ & $0: 00: 01,685$ \\
\hline 7 & $18: 12: 42,447$ & Not Detected & & $18: 12: 43,882$ & 0:00:01,435 \\
\hline 8 & $18: 12: 51,698$ & Not Detected & & $18: 12: 54,022$ & $0: 00: 02,324$ \\
\hline 9 & $18: 13: 02,571$ & Not Detected & & 18:13:04,162 & 0:00:01,591 \\
\hline 10 & $18: 13: 11,650$ & $18: 13: 14,057$ & $0: 00: 02,407$ & $18: 13: 13,288$ & $0: 00: 01,638$ \\
\hline 11 & $18: 13: 25,848$ & Not Detected & & $18: 13: 27,484$ & $0: 00: 01,636$ \\
\hline 12 & $18: 13: 36,830$ & $18: 13: 44,440$ & $0: 00: 07,610$ & $18: 13: 38,638$ & 0:00:01,808 \\
\hline 13 & $18: 13: 55,550$ & Not Detected & & $18: 13: 57,967$ & $0: 00: 02,417$ \\
\hline 14 & $18: 14: 08,577$ & $18: 14: 23,450$ & $0: 00: 14,873$ & 18:14:10,946 & $0: 00: 02,369$ \\
\hline 15 & $18: 14: 25,003$ & Not Detected & & $18: 14: 26,593$ & $0: 00: 01,590$ \\
\hline
\end{tabular}




\begin{tabular}{|cc|cc|}
\hline Average & 0:00:08,073 & Average & $0: 00: 01,861$ \\
\hline Min & $0: 00: 02,407$ & Min & $0: 00: 01,300$ \\
\hline Max & $0: 00: 14,873$ & Max & $0: 00: 02,417$ \\
\hline $\begin{array}{c}\text { Detection } \\
\text { rate }\end{array}$ & $53,33 \%$ & $\begin{array}{c}\text { Detection } \\
\text { rate }\end{array}$ & $100,00 \%$ \\
\hline
\end{tabular}

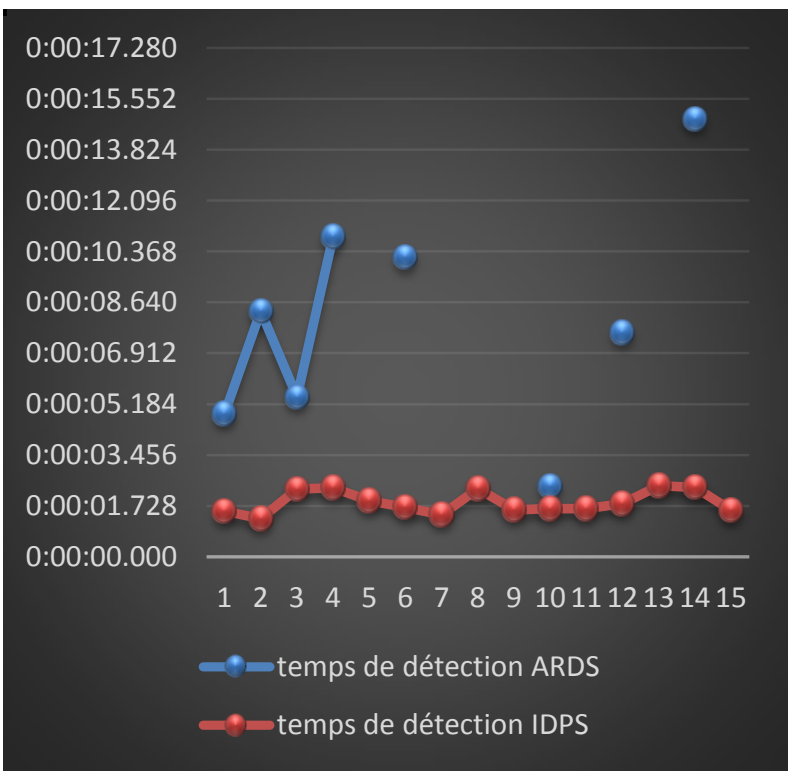

Fig 3: Evolution of the detection time of an attack HIDPS/ARD

Of course, this detection time may vary depending on:

- The physical characteristics of our simulation system workstations, network cards, Switch ...

- Network saturation at the time of the attack

- The number of attacks

- The duration between attacks

- The number and nature of security rules

- etc.

But, nevertheless, we note that:

- The threat detection rate HIDPS is $100 \%$ at the time the ARD is only $53.3 \%$

- The detection time of the HIDPSS is significantly better than that of ARD

Thus, we discover that an embedded system is not in all cases the fastest system. But it depends of security purposes.

\subsection{NIDPS/ARD System}

\subsubsection{Diagram of the simulation}

In this case we pair an NIDPSS with an ARD as below:

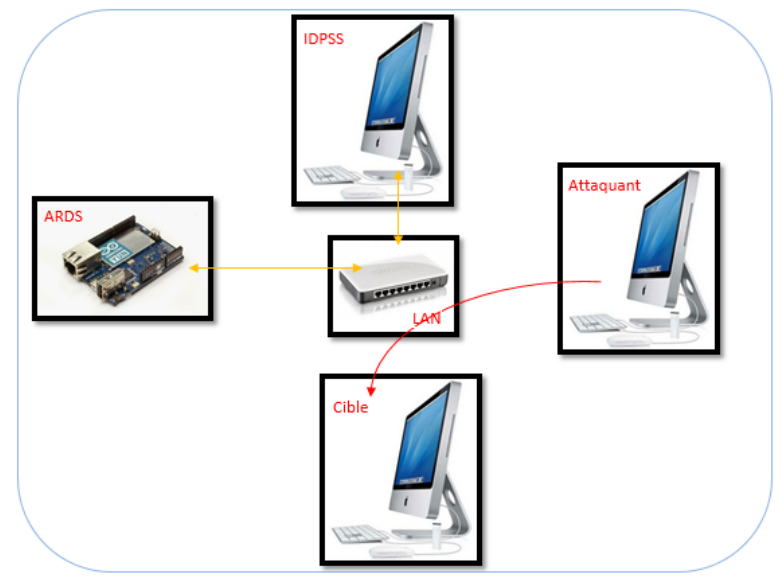

Fig 4: Case NIDPS/ARD

\subsubsection{Evaluation of the detection time}

We carry out a series of attacks on our detection system to assess its response time to an attack. Thus we get the results below.

Table 2. Summary of different detection time - NIDPS / ARD

\begin{tabular}{|c|c|c|c|c|c|}
\hline $\begin{array}{l}\text { Attack } \\
\text { Number }\end{array}$ & $\begin{array}{l}\text { Attack } \\
\text { Instant }\end{array}$ & $\begin{array}{c}\text { ARD } \\
\text { Detection } \\
\text { Instant }\end{array}$ & $\begin{array}{c}\text { Detection } \\
\text { Time ARD } \\
\text { (ms) }\end{array}$ & $\begin{array}{c}\text { IDPS } \\
\text { Detection } \\
\text { Instant }\end{array}$ & $\begin{array}{c}\text { Detection } \\
\text { Time IDPS } \\
\text { (ms) }\end{array}$ \\
\hline 1 & $18: 19: 42,000$ & $18: 19: 52,126$ & $0: 00: 10,126$ & $18: 19: 52,983$ & $0: 00: 10,983$ \\
\hline 2 & $18: 20: 04,884$ & $18: 20: 25,297$ & $0: 00: 20,413$ & $18: 20: 26,446$ & $0: 00: 21,562$ \\
\hline 3 & $18: 20: 36,928$ & $\begin{array}{c}\text { Not } \\
\text { Detected }\end{array}$ & & $\begin{array}{c}\text { Not } \\
\text { Detected }\end{array}$ & \\
\hline 4 & $18: 21: 04,352$ & $18: 21: 08,428$ & $0: 00: 04,076$ & $18: 21: 10,346$ & $0: 00: 05,994$ \\
\hline 5 & $18: 21: 23,728$ & $\begin{array}{c}\text { Not } \\
\text { Detected }\end{array}$ & & $\begin{array}{c}\text { Not } \\
\text { Detected }\end{array}$ & \\
\hline 6 & $18: 21: 41,809$ & $18: 21: 46,160$ & $0: 00: 04,351$ & $18: 21: 47,053$ & $0: 00: 05,244$ \\
\hline 7 & $18: 22: 04,226$ & $18: 22: 04,624$ & $0: 00: 00,398$ & $18: 22: 04,390$ & $0: 00: 00,164$ \\
\hline 8 & $18: 22: 22,634$ & $\begin{array}{c}\text { Not } \\
\text { Detected }\end{array}$ & & $\begin{array}{c}\text { Not } \\
\text { Detected }\end{array}$ & \\
\hline 9 & $18: 22: 41,651$ & $18: 22: 46,293$ & $0: 00: 04,642$ & $18: 22: 48,025$ & $0: 00: 06,374$ \\
\hline 10 & $18: 23: 02,000$ & $18: 23: 03,215$ & $0: 00: 01,215$ & $18: 23: 02,221$ & $0: 00: 00,221$ \\
\hline 11 & $18: 23: 21,000$ & $18: 23: 33,712$ & $0: 00: 12,712$ & $18: 23: 34,670$ & $0: 00: 13,670$ \\
\hline 12 & $18: 23: 35,674$ & $18: 23: 49,072$ & $0: 00: 13,398$ & $18: 23: 50,895$ & $0: 00: 15,221$ \\
\hline 13 & $18: 23: 56,002$ & $18: 24: 03,415$ & $0: 00: 07,413$ & $18: 24: 05,091$ & 0:00:09,089 \\
\hline 14 & $18: 24: 11,773$ & $18: 24: 15,752$ & $0: 00: 03,979$ & $18: 24: 17,276$ & $0: 00: 05,503$ \\
\hline \multirow[t]{5}{*}{15} & $18: 24: 25,143$ & $18: 24: 30,162$ & $0: 00: 05,019$ & $18: 24: 31,456$ & $0: 00: 06,313$ \\
\hline & & Average & $0: 00: 07,312$ & Average & $0: 00: 08,361$ \\
\hline & & Min & $0: 00: 00,398$ & Min & $0: 00: 00,164$ \\
\hline & & Max & $0: 00: 20,413$ & Max & $0: 00: 21,562$ \\
\hline & & $\begin{array}{l}\text { Detection } \\
\text { rate }\end{array}$ & $80,00 \%$ & $\begin{array}{l}\text { Detection } \\
\text { rate }\end{array}$ & $80,00 \%$ \\
\hline
\end{tabular}




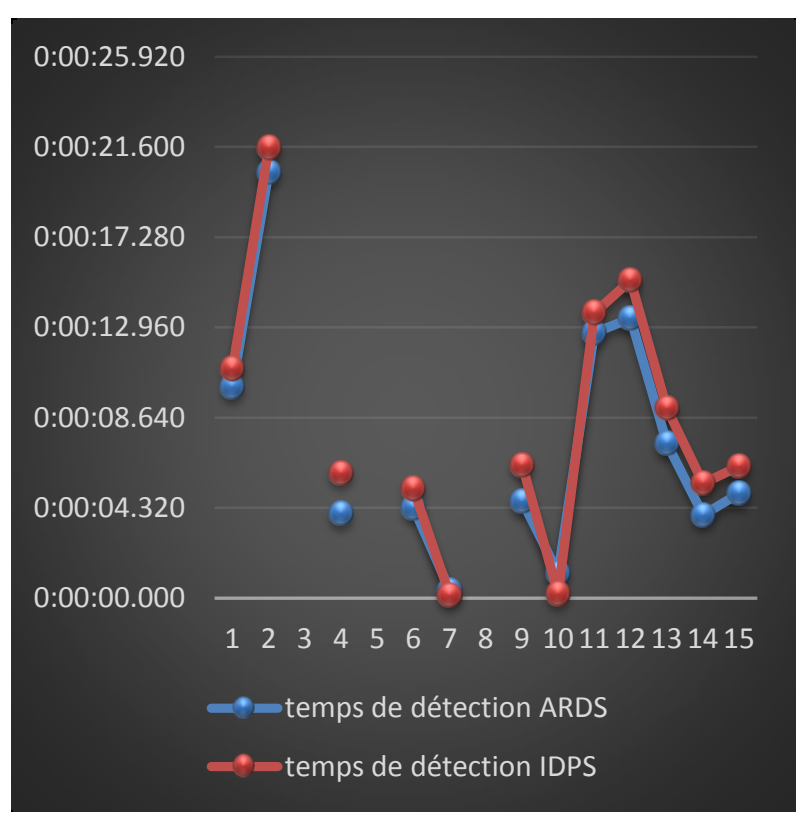

Fig 5: Evolution of the detection time of an attack NIDPSS/ARD-S

Of course, this detection time may vary according to the same conditions mentioned in the previous section.

But, nevertheless, we note that:

- The detection rates of ARD and NIDPS are not $100 \%$

- The ARD detection time is on average faster than the NIDPSS

Thus, we can notice that unlike the previous case, the embedded system has better performance.

\section{CONCLUSION AND FURTHER WORK}

In this paper, we proposed hybrid security architecture based on a distributed approach of NIDPS, HIDPS, KIDPS and Arduino Board according to spatial and semantic distributions based on detection method.

We noted that the embedded system has, in the case of an analysis of the network, the fastest response time, when the software system prevails in the case of the direct protection of a host. Nevertheless, the software system offers opportunities for more advanced prevention. These results support the importance of our probes combination and distribution in the design of our security architecture. A distribution that covers various scenarios and ensures in all cases the best response time.

As further work, we can study the possibility to create with Arduino Boards a Proxy system to improve the prevention of the embedded system.

\section{REFERENCES}

[1] Open Information Security Foundation. « Getting Started With Suricata ». OISF, 2011

[2] Karen Scarfone, Peter Mell. "Guide to Intrusion Detection and Prevention Systems IDPS". NIST. US Departement of Commerce. 2007

[3] Daniel Guinier. "Sécurité et qualité des systèmes d'information - Approche systémique". Masson. 1992

[4] Boriana Ditcheva, Lisa Fowler. "Signature-based Intrusion Detection". University of North Carolina at Chapel Hill. 2005

[5] Martin Roesch, Chris Green, Sourcefire, Inc. "SNORT User's Manual 2.9.0”. The Snort Project. 2010

[6] WINPCAP documentation. Copyright (c) 2002-2005 Politecnico di Torino Dsfg

[7] Rachid Guerraoui, Lu'is Rodrigues, "Introduction to reliable distributed programming", Springer-Verlag, August 24, 2005.

[8] Web Site: Arduino - http://www.arduino.cc/.

[9] Y.SENHAJI, "Network Security: Distributed Agents Approach', International Journal of Emerging Trends \& Technology in Computer Science (IJETTCS), Volume 01, Issue 02, July-August 2012

[10] Y.SENHAJI, H.MEDROMI, "Network Security: ARDUINO Yun Based IDS", International Journal of Emerging Trends \& Technology in Computer Science (IJETTCS), Volume 4, Issue 4, July - August 2015 